\title{
Transbronchial lung cryobiopsy (TBLC) at a Tertiary Referral Hospital: A two-year experience
} \author{
Camporesi (10* $^{9}$ \\ ${ }^{1}$ Research Coordinator, TEAMHealth Anesthesia Research Institute, Tampa, FL, USA \\ ${ }^{2}$ Student, University of South Florida, Tampa, FL, USA \\ ${ }^{3}$ Assistant Professor, Pulmonary, Critical Care \& Sleep Medicine, University of South Florida, Tampa, FL, USA \\ ${ }^{4}$ Resident, Department of Pathology, Tampa General Hospital, Tampa, FL, USA \\ ${ }^{5}$ Fellow, Pulmonary, Critical Care \& Sleep Medicine, University of South Florida, Tampa, FL, USA \\ ${ }^{6}$ Division Director, Pulmonary, Critical Care \& Sleep Medicine, University of South Florida, Tampa, FL, USA \\ ${ }^{7}$ Regional Medical Director, TEAMHealth Anesthesia, Tampa, FL, USA \\ ${ }^{8}$ Chief of Anesthesia, Tampa General Hospital, Tampa, University of South Florida, Tampa, FL, USA \\ ${ }^{9}$ Director of Research, TEAMHealth Anesthesia Research Institute, Tampa, FL, USA \\ ${ }^{10}$ Professor Emeritus, University of South Florida, Tampa, FL, USA
}

Prachiti Dalvi ${ }^{1}$, Gabriella Kretz ${ }^{2}$, Stephen Clum ${ }^{3}$, Michael Markow ${ }^{4}$, David DiNuoscio ${ }^{5}$, Mark Rumbak ${ }^{6}$, Devanand Mangar ${ }^{7,8}$ and Enrico

\begin{abstract}
Introduction: Interstitial lung disease is difficult to diagnose because radiological imaging can often be inconclusive. Surgical lung biopsies are the standard for diagnosis, but are associated with complications and morbidity because they are invasive. Transbronchial lung cryobiopsies (TBLC) are less invasive and allow for the collection of large pieces of tissue by cryoadhesion and, thus, are better diagnostic tools for this group of diffuse parenchymal lung diseases, especially for differentiating between UIP (usual interstitial pneumonia) and NSIP (non-specific interstitial pneumonia).
\end{abstract}

Materials and methods: After IRB approval, we conducted a retrospective chart review of patients who had undergone TBLC to diagnose interstitial lung disease at Tampa General Hospital from 2012 to 2014.

Results: Only 5 of the 74 patients had a diagnosis of favorable or definitive UIP. Four out of our 74 patients (5.4\%) experienced a pneumothorax in our study population. Of the 4 patients who experienced pneumothorax, only 3 patients required chest tubes. Our transbronchial cryobiopsy technique also led to more conclusive histological imaging and there was no incidence of significant bleeding.

Discussion: Our methodology was associated with only minor bleeding, managed with epinephrine and did not require thrombin application. No Fogarty catheters needed to be inserted endobronchially in the vicinity of the bronchoscope to stem the bleeding. Overall, our modified protocol for transbronchial cryobiopsies presents a refined approach to diagnosing interstitial lung disease while significantly reducing the risk of pneumothorax and producing more compelling histology.

\section{Introduction}

Interstitial lung disease is challenging to diagnose in the event that radiological imaging alone is inconclusive. Surgical lung biopsy remains the gold standard; however, because of its invasive nature, it is associated with complications and increased risk of mortality [1,2]. More recently, a new method of biopsy called transbronchial lung cryobiopsy (TBLC) has been utilized to diagnose interstitial lung disease [3].

Transbronchial lung cryobiopsies (TBLC) allow for the collection of large pieces of tissue by cryoadhesion and, thus, represents a new diagnostic tool for this group of fibrotic diffuse parenchymal lung diseases [3-5]. This method has been shown to produce a more conclusive histologic image, when compared to needle core biopsies, in a few studies but results from these papers are not comparable because of various sampling techniques and sedation regimens (Figure 1) [6].

This retrospective chart review was approved by the University of South Florida's Institutional Review Board (Pro00021732). Data were collected from patient demographics and medical records of patients who met the inclusion and exclusion criteria from January 31, 2013 to May 22, 2015. We collected the following information from patient charts: incidence of pneumothorax, placement of chest tube, incidence of transbronchial hemorrhage, post-TBLC intubation, incidence of transbronchial fistula, transfusion or blood products, and duration of hospital stay. We reported categorical variables as percentages of total subjects.

\section{Materials and methods}

In the past two years, we have utilized TBLC at Tampa General

Correspondence to: Enrico M. Camporesi, MD, Tampa General Circle, Suite A327, Tampa, FL 33606, USA, Tel: 813-844-4434, Fax: 813-844-4467; E-mail: ecampore@health.usf.edu

Received: December 02, 2016; Accepted: December 24, 2016; Published: December 28, 2016 


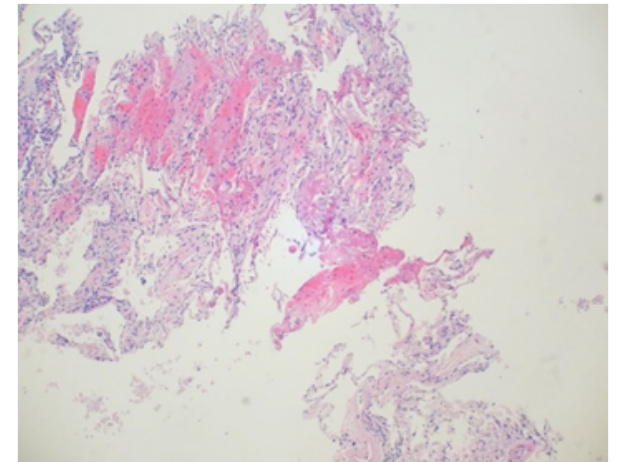

Figure 1. Needle core biopsies of the lung produce greater surgical and processing artifact in tissues than those taken using the cryobiopsy procedure. Alveoli tend to become collapsed, and intra-alveolar cells and debris are more likely to be washed out during tissue processing. Additionally, toward the top-left there is artefactual hemorrhage and fibrin. Together, these morphological alterations can obscure the histopathological findings within the tissue. Needle core biopsy, H\&E, 10x objective magnification.

Hospital for the diagnosis of interstitial lung disease, with general anesthesia. These patients are usually referred to us by primary care physicians from a large surrounding area. Patients were seen by the University of South Florida's Pulmonary Division with diagnosis of diffuse lung disease. Cryobiopsy was performed for tissue diagnosis. After induction of general anesthesia and tracheal intubation, we use a short freezing cycle (3-4 seconds) and by radiological assessment we verify that the tip of the cryoprobe (ERBE, Tübingen, Germany) is retracted at least $1.5 \mathrm{~cm}$ away from the pleura to minimize the risk of pneumothorax. We also wedge the scope in the sub-segment from which the biopsy tissue is going to be obtained and infuse lidocaine (2\%; $2 \mathrm{~mL}$ per biopsy) with epinephrine $(1: 100,000 ; 1-2 \mathrm{~mL})$ prior to obtaining tissue biopsy to minimize the risk of bleeding. With these notable differences in techniques, our experience has been of very minimal bleeding complications; hence, we do not insert a Fogarty catheter in the vicinity of the bronchoscope to control bleeding postbiopsy. Lastly, we rapidly remove the frozen sample on the cryoprobe tip as well as the bronchoscope directly from the endotracheal tube, the endotracheal tube being kept firmly in place by an assistant. We present patient outcomes and complications when using our TBLC protocol and compare them to current literature.

\section{Results}

In general, biopsies are not performed on patients with a clinical history and radiological findings compatible with a diagnosis of UIP as lung biopsy in this patient population has been associated with increased mortality precipitated by the procedure [2]. If CAT scans and clinical history are not diagnostic for UIP, lung biopsy is necessary for diagnosis of the specific interstitial lung disease. Given the increased diagnostic yield observed with cryobiopsies, our institution has begun to employ this method to obtain lung tissue. At our institution, only 5 of the $74(6.8 \%)$ of the cryobiopsy samples were diagnosed to be UIP, indicating that the predictive value of CAT scans is high. Figures 2-4 represent different histological patterns observed from TBLC sampling.

Four out of our 74 patients (5.4\%) experienced a pneumothorax in our study population, with only one other study group reporting an incidence of pneumothorax less than ours [8]. Of the 4 patients who experienced pneumothorax, only 3 patients required chest tubes. Additionally, our methodology was associated with only minor bleeding, managed with epinephrine $(1: 100,000 ; 1-2 \mathrm{~mL})$. None of the patients in our study population required the insertion of Fogarty catheters pre- or post- procedure. The median length of stay in the hospital after our procedure was 0 days with only one patient needing to stay for 11 days: this last patient was admitted to the hospital after an inconclusive bronchoscopy to provide care for bilateral pleural effusion and pneumonia, which resolved. She was discharged in stable condition. Two patients in our study population died 30 and 4 days, respectively, after the cryobiopsy but the procedure was likely not contributory: one death was related to non-pulmonary complications from prostate cancer and the second death was due to continuation of deterioration from prior renal and cardiac insufficiency leading to admission to the ICU for continuing cardiac deterioration (ejection fraction of less than 30\%) despite maximal therapy.

\section{Discussion}

Interstitial lung disease includes a broad differential of clinical entities [7]. Accurate diagnosis of a specific interstitial lung disease requires detailed clinical, occupational, environmental histories, as well as temporal evaluation of radiological and clinical symptoms and a clear histological picture. Tissue analysis can be misleading if the structure of the tissue is modified by forces such as needle biopsies (Figure 1). Despite methodical clinical and radiological evaluation, definitive diagnosis of ILD often requires tissue analysis. Cryobiopsy techniques maintain the structural integrity of pulmonary tissues to a greater extent; however, pathologists report feeling less confident in their interpretation of TBLC alone when compared to surgical lung biopsy (Figure 2) [9]. The challenge of differentiating between UIP and NSIP is evident by comparing Figures 3 and 4, as detailed in the

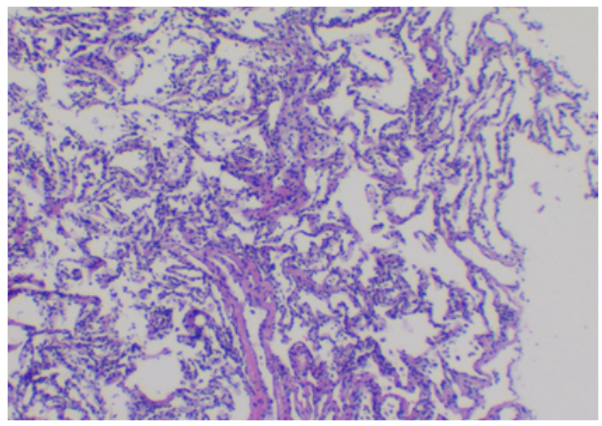

Figure 2. Normal alveolar tissue is seen with thin, delicate alveolar septa. Multiple (4 or more) fragments of tissue typically yield at least one with an abundant amount of alveolar tissue as seen here. Cryobiopsy, lung, H\&E, 40x.

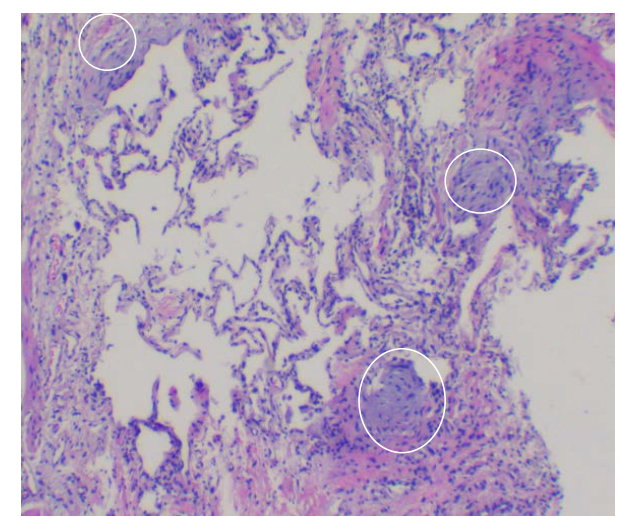

Figure 3. Usual interstitial pneumonia (UIP) can be seen with a heterogeneous pattern radiographically, grossly, and microscopically. Within this field are unaffected alveoli (center), interstitial fibrosis, and minimal lymphocytosis (right side), and three characteristic fibroblastic foci (circled). True fibroblastic foci are distinctive for interstitial lung diseases. Usual interstitial pneumonia has patchy distribution of fibroblastic foci. Fibroblastic foc have spindled fibroblasts oriented parallel to the alveolar septa, with interstitial granular, basophilic ground substance. Cryobiopsy, lung, H\&E, 40x. 
Table 1. Comparison of pneumothorax incidence and bleeding incidence with current literature.

\begin{tabular}{|c|c|c|c|c|c|}
\hline Study [Reference Number] & Study design & $\mathbf{n}$ & Incidence of Pneumothorax & Incidence of Bleeding & Diagnostic Yield \\
\hline Casoni et al. 2014 [6] & Prospective & 69 & $27.5 \%$ & $1.4 \%$ & $93 \%$ \\
\hline Hagmeyer et al. 2015 [11] & Retro- \& Prospective & 51 & $26 \%$ & $78 \%$ & $75 \%$ \\
\hline Hernandez-Gonzalez et al. 2015 [12] & Retrospective & 33 & $12 \%$ & $30 \%$ & $79 \%$ \\
\hline Tomassetti et al. 2015 [10] & Retrospective & 91 & $33 \%$ & $0 \%$ & $91 \%$ \\
\hline Ussavarungsi et al. 2016 [8] & Retrospective & 74 & $1.4 \%$ & $22 \%$ & $51 \%$ \\
\hline Current study & Retrospective & 74 & $5.4 \%$ & $0 \%$ & $88 \%$ \\
\hline
\end{tabular}

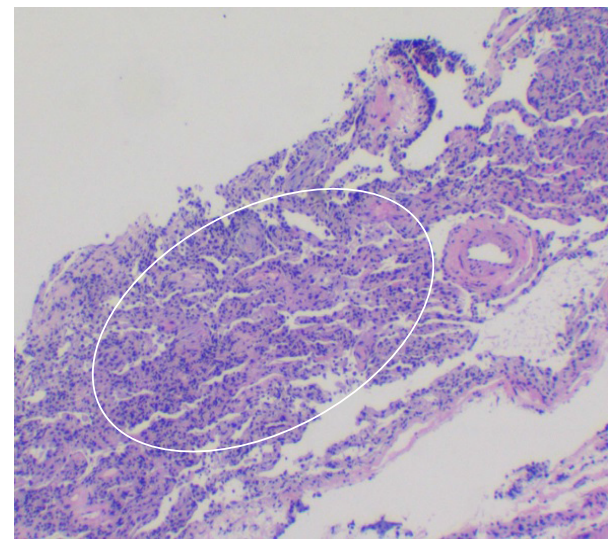

Figure 4. Non-specific interstitial pneumonia (NSIP) has a diffuse pattern (circled) of involvement. This image represents cellular non-specific interstitial pneumonia, which has diffuse lymphoplasmacytic interstitial inflammation within the alveolar septa. There is hyperplasia of type II pneumocytes within the alveolar spaces. Toward the top-left, there are two fibroblastic foci comprised of spindled fibroblasts aligned parallel to alveolar septa They have characteristic granular, basophilic ground substance. Cryobiopsy, lung, H\&E, 40x.

figure legends. Though challenging to make, this distinction can afford significant treatment advantage for UIP patient [7].

Overall, our modified protocol for transbronchial cryobiopsies presents a refined approach to diagnosing interstitial lung disease while significantly reducing the risk of procedural complications. The length of our freezing cycle and probe positioning resulted in a much lower incidence of pneumothorax than is currently reported in the literature. The diagnostic yield of the biopsy (88\%) did not appear to be compromised by the shortened freeze time as indeterminate results were obtained in only 9 of the 74 biopsies (12\%). The shortened freeze time (3-4 seconds) and pre-treatment with epinephrine resulted in much less bleeding complications post-biopsy. No patients required interventions beyond use of epinephrine or thrombin to control bleeding. All probes were retracted $2-3 \mathrm{~cm}$ from the pleural surface using fluoroscopic guidance before freezing.

Two recent studies published by Ussavarungsi et al. (2016) and Ravaglia (2016) evaluate the current literature for transbronchial cryobiopsy in patients with diffuse parenchymal lung diseases [8,9]. As seen in the table in the Ussavarungsi publication (2016), most literature in this area consists of retrospective studies. Though there are notable differences in incidence of pneumothorax and bleeding throughout the literature, comparisons between these studies is challenging given the heterogeneity of individual study populations and possibly different interpretation of bleeding intensity from each author (Table 1). We believe the wide variation in incidence of pneumothorax and bleeding is primarily due to the differences in cryobiopsy protocols and techniques at each institution.

In conclusion, the modified technique described for transbronchial cryobiopsies should facilitate further use of this technique in the characterization of interstitial lung diseases not classifiable by clinical history combined with radiological and serological evaluation. Accurate diagnosis of these lung disorders will guide treatment of the underlying lung diseases. Furthermore, since surgical lung biopsy will not be required, decreased cost in the overall diagnosis and management of these patients would be expected.

\section{Funding}

Supported by internal funding from the TEAMHealth Anesthesia Research Institute.

\section{Previous presentation}

This research was previously presented in part at the International Anesthesia Research Society Meeting in San Francisco, CA, May 21-24, 2016.

\section{Author contributions}

Prachiti H. Dalvi was involved in data analysis, manuscript writing, and regulatory oversight. Gabriella Kretz helped with data collection. Dr. Stephen Clum performed data analysis and helped write the manuscript. Dr. Michael Markow helped with imaging and data analysis. Dr. David Dinuoscio assisted with data collection. Dr. Mark Rumbak helped with data analysis. Dr. Devanand Mangar helped with protocol design and data analysis. Dr. Enrico Camporesi was involved in protocol design, regulatory approvals, data collection, data analysis, and manuscript writing. Dr. Enrico Camporesi served as the Principal Investigator for this study.

\section{References}

1. Kreider ME, Hansen-Flaschen J, Ahmad NN, Rossman MD, Kaiser LR, et al. (2007) Complications of video-assisted thoracoscopic lung biopsy in patients with interstitial lung disease. Ann Thorac Surg 83: 1140-1144. [Crossref]

2. Utz JP, Ryu JH, Douglas WW, Hartman TE, Tazelaar HD, et al. (2001) High short-term mortality following lung biopsy for usual interstitial pneumonia. Eur Respir J 17: 175179. [Crossref]

3. Babiak A, Hetzel J, Krishna G, Fritz P, Moeller P, et al. (2009) Transbronchial cryobiopsy: a new tool for lung biopsies. Respiration 78: 203-208. [Crossref]

4. Yarmus L, Akulian J, Gilbert C, Illei P, Shah P, et al. (2013) Cryoprobe transbronchia lung biopsy in patients after lung transplantation: a pilot safety study. Chest 143: 621626. [Crossref]

5. Pajares V, Torrego A, Puzo C, Lerma E, Gil De Bernabé MA, et al. (2010) [Transbronchial lung biopsy using cryoprobes]. Arch Bronconeumol 46: 111-115. [Crossref]

6. Casoni GL, Tomassetti S, Cavazza A, Colby TV, Dubini A, et al. (2014) Transbronchial lung cryobiopsy in the diagnosis of fibrotic interstitial lung diseases. PLoS One 9: e86716. [Crossref]

7. Travis WD, Costabel U, Hansell DM, King TE Jr, Lynch DA, et al. (2013) An Official American Thoracic Society/European Respiratory Society Statement: Update of the International Multidisciplinary Classification of the Idiopathic Interstitial Pneumoniasm. Am J Respir Crit Care Med 188: 733-748. [Crossref]

8. Ussavarungsi K, Kern RM, Roden AC, Ryu JH, Edell ES (2016) Transbronchia Cryobiopsy in Diffuse Parenchymal Lung Disease: Retrospective Analysis of 74 Cases. Chest [Crossref] 
Dalvi P (2016) Transbronchial lung cryobiopsy (TBLC) at a Tertiary Referral Hospital: A two-year experience

9. Ravaglia C, Bonifazi M, Wells AU, Tomassetti S, Gurioli C, et al. (2016) Safety and Diagnostic Yield of Transbronchial Lung Cryobiopsy in Diffuse Parenchymal Lung Diseases: A Comparative Study versus Video-Assisted Thorascopic Lung biopsy and a Systematic Review of the Literature. Respiration 91: 215-227. [Crossref]

10. Tomassetti S, Wells AU, Costabel U, Cavazza A, Colby TV, et al. (2016) Bronchoscopic Lung Cryobiopsy Increases Diagnostic Confidence in the Multidisciplinary Diagnosis of Idiopathic Pulmonary Fibrosis. Am J Respir Crit Care Med 193: 745-752. [Crossref]
11. Hagmeyer L, Theegarten D, Wohlschläger J, Treml M, Matthes S, et al. (2016) The role of transbronchial cryobiopsy and surgical lung biopsy in the diagnostic algorithm of interstitial lung disease. Clin Respir J 10: 589-595. [Crossref]

12. Hernández-González F, Lucena CM, Ramírez J, Sánchez M, Jimenez MJ, et al (2015) Cryobiopsy in the diagnosis of diffuse interstitial lung disease: yield and costeffectiveness analysis. Arch Bronconeumol 51: 261-267. [Crossref]

Copyright: @2016 Dalvi P. This is an open-access article distributed under the terms of the Creative Commons Attribution License, which permits unrestricted use, distribution, and reproduction in any medium, provided the original author and source are credited. 